\title{
Análise Fatorial para Avaliação dos Questionários de Satisfação do Curso de Estatística de uma Instituição Federal
}

\section{Factorial Analysis for the Evaluation of Satisfaction Questionnaires of the Statistics Course of a Federal Institution}

\author{
${ }^{1}$ Jhessica Letícia Kirch, ${ }^{2}$ Kuang Hongyu, ${ }^{3}$ Fabiane de Lima Silva, ${ }^{4}$ Carlos Tadeu dos Santos Dias \\ ${ }^{1}$ Graduanda no Departamento de Estatística, Instituto de Ciências Exatas e da Terra - ICET da Universidade \\ Federal de Mato Grosso - UFMT (jhessicakirch@gmail.com) \\ ${ }^{2}$ Professor Adjunto do Departamento de Estatística, ICET/UFMT (prof.kuang@gmail.com) \\ ${ }^{3}$ Professora Adjunta do Centro de Ciências Agrárias, Ambientais e Biológicas-Universidade Federal do \\ Recôncavo da Bahia (fabianezte@yahoo.com.br) \\ ${ }^{4}$ Professor Titular do Departamento de Ciências Exatas/ESALQ-USP, Avenida Pádua Dias, 11, CEP 13418-900, \\ Piracicaba, SP, Brasil. (ctsdias@usp.br)
}

\begin{abstract}
RESUMO: O presente estudo buscou construir um instrumento de pesquisa de satisfação de alunos de um curso de educação superior e investigar a estrutura fatorial deste questionário. A coleta dos dados foi realizada por meio de um questionário fechado, baseado na escala Likert, com respostas variando em cinco níveis de satisfação. A amostra estratificada foi composta por 50 estudantes do Departamento de Estatística da Universidade Federal de Mato Grosso - UFMT. Foi utilizada a Análise Fatorial Exploratória, que sugeriu o agrupamento das 19 variáveis do questionário em quatro fatores distintos, que proporcionaram maior homogeneidade entre as variáveis dentro de cada grupo: Professores de outros departamentos que ministram aula aos alunos de Estatística, Instalações físicas e estruturais, Professores do Departamento de Estatística, e Desempenho do Aluno (Auto avaliação).O total dos fatores explicou100\% da variação total, o primeiro fatorexplicou $27 \%$, o segundo, $26 \%$, o terceiro, $24 \%$ e o quarto, $23 \%$. Em pesquisas futuras poderá ser utilizado o novo questionário, organizado nas dimensões identificadas por meio da análise fatorial.

Palavras-chave: Análise multivariada; Satisfação na educação; Análise fatorial exploratória.
\end{abstract}

ABSTRACT: The aim of this study is to construct a survey instrument for the satisfaction of students of a higher education course and to investigate a factorial structure of this questionnaire. Data were collected through a closed questionnaire based on a Likert scale, with responses varying in five levels of satisfaction. The stratified sample consisted of 50 students from the Statistics Department of the Federal University of Mato Grosso UFMT. An Exploratory Factor Analysis was performed, which suggests the grouping of the 19 variables of the questionnaire into four different dimensions that providing greater homogeneity among variables within each group.: The teams from other departments that provide classes for students of Statistics, Physical and Structural Installations, Teachers of the Department of Statistics and Student Performance (Self-Assessment). The total factor accounts for $100 \%$ of the total variance. The first factor explains $27 \%$, the second, $26 \%$, the third, $24 \%$ and the fourth, $23 \%$. In terms of the use of the new questionnaire, organized in dimensions identified through the factorial analysis.

Keywords: Multivariate Analysis; Satisfaction in education; Exploratory factor analysis.

\section{INTRODUÇÃO}

No campo da educação, avaliar a qualidade de um curso por meio da satisfação do aluno significa avaliar as percepções de um indivíduo que é, tanto passivo, bem como um agente ativo na construção de uma educação de qualidade. Como consequência disso, a satisfação de um aluno é dada por meio das suas percepções e expectativas em relação à estrutura física e tecnológica do curso, aos serviços prestados pelos professores e técnicos, como também pelo seu próprio desempenho, que resulta em uma condição determinante para a qualidade da instituição. 
Os questionários de satisfação, como instrumentos de pesquisa, possibilitam conhecer a opinião de um grupo específico em relação a um produto ou serviço de interesse. Com isso, é possível avaliar a importância dada a cada item explorado. Para isso, o questionário deve ser elaborado e validado de maneira adequada. Um questionário é composto por um número de questões, ou itens, frequentemente agrupados em fatores, que buscam traduzir um conceito geral. Assim, para validar um questionário, faz-se necessário verificar se os itens são suscetíveis a serem agrupados. (HOSS, CATEN, 2010). A Análise Fatorial Exploratória (AFE), quando utilizada para validação de um instrumento de pesquisa, possibilita a divisão do questionário em fatores e a identificação das questões mais representativas do instrumento.

A AFE é uma técnica estatística que estuda correlações entre um grande número de variáveis agrupando-as em fatores. Essa técnica permite a redução de dados, identificando as variáveis mais representativas ou criando um novo conjunto de variáveis, bem menor que o original (HAIR Jr. et al., 2005).

Nesse contexto, o presente trabalho teve por objetivo construir e validar um instrumento de avaliação da satisfação dos alunos do Departamento de Estatística da Universidade Federal de Mato Grosso (UFMT) utilizando a técnica da análise fatorial exploratória.

\section{MATERIAIS E MÉTODOS}

A pesquisa foi realizada com 50 estudantes do curso de Graduação em Estatística da Universidade Federal de Mato Grosso (UFMT), sendo 23 do gênero feminino (46\% do total) e 27 do masculino (54\%).

A amostragem foi feita em duas etapas. Na primeira etapa, dividiu-se o total de alunos matriculados no curso em 4 subgrupos de acordo com o ano de estudo que cursava, em seguida foi aplicada uma amostragem aleatória simples em cada subgrupo, caracterizando assim uma amostragem estratificada. Desta forma, 24\% dos respondentes eram do primeiro ano, $36 \%$ do segundo ano, $28 \%$ do terceiro ano e $12 \%$ do quarto ano do curso.

Como instrumentos de coletas de dados utilizou-se um questionário estruturado fechado contendo 20 questões, sendo 19 questões sobre a satisfação do aluno e uma questão sobre seu gênero. Com o intuito de avaliar a satisfação dos alunos para cada um dos itens, as respostas tinham por características serem objetivas de concordância, utilizando para isso a escala de Likert, com cinco opções de resposta, variando de muito insatisfeito a muito satisfeito.

As 19 questões sobre a satisfação dos alunos que compõem o questionário são apresentadas no Quadro 1, bem como suas abreviaturas utilizadas no decorrer deste trabalho.

\section{$O$ indice $K M O$}

O primeiro passo durante a implementação de AFE (pressupostos) é verificar se a aplicação da análise fatorial tem validade para as variáveis escolhidas, sendo justificado pela pouca quantidade de respondentes da pesquisa. Para isso, dois métodos de avaliação são mais comumente utilizados, o critério de Kaiser-Meyer-Olkin (KMO) e o Teste de Esfericidade de Bartlett (DZIUBAN; SHIRKEY, 1974; DAMÁSIO, 2012).

O índice de KMO, também conhecido como índice de adequação da amostra, é um teste estatístico que sugere a proporção de variância dos itens que pode estar sendo explicada por uma variável latente, tal índice indica o quão adequada é a aplicação da AFE para o conjunto de dados (LORENZO-SEVA; TIMMERMAN; KIERS, 2011; HAIR et al., 2005; DAMÁSIO, 2012). O KMO é calculado por meio do quadrado das correlações totais dividido pelo quadrado das correlações parciais, das variáveis analisadas, cuja expressão é (DZIUBAN; SHIRKEY, 1974). 
Kirch, J.L.; Hongyu, K; Silva, F. de L;Dias, C. T.; Análise Fatorial para Avaliação dos Questionários de Satisfação do Curso de Estatística de uma Instituição Federal. E\&S - Engineering and Science, (2017), 6:1.

Quadro 1. Itens que compuseram o questionário.

\begin{tabular}{|c|c|}
\hline Salas & Qual seu grau de satisfação em relação às salas de aulas? \\
\hline Laboratório & Qual seu grau de satisfação em relação ao laboratório de informática? \\
\hline Biblioteca & Qual seu grau de satisfação em relação à biblioteca? \\
\hline Centro & Qual seu grau de satisfação em relação ao centro acadêmico? \\
\hline Departamento & Qual seu grau de satisfação em relação ao departamento? \\
\hline Recursos & Qual seu grau de satisfação em relação aos recursos tecnológicos? \\
\hline Bolsas & $\begin{array}{l}\text { Qual seu grau de satisfação em relação à disponibilidade de bolsas (monitoria, } \\
\text { PIBIC, extensão, outras? }\end{array}$ \\
\hline Disponib_Est & $\begin{array}{l}\text { Qual seu grau de satisfação em relação à disponibilidade fora de sala de aula dos } \\
\text { professores do Departamento de Estatística? }\end{array}$ \\
\hline Dominio_Est & $\begin{array}{c}\text { Qual seu grau de satisfação em relação ao domínio do conteúdo pelos professores } \\
\text { do Departamento de Estatística? }\end{array}$ \\
\hline Pontual_Est & $\begin{array}{c}\text { Qual seu grau de satisfação em relação à pontualidade dos professores do } \\
\text { Departamento de Estatística? }\end{array}$ \\
\hline Mat_Est & $\begin{array}{l}\text { Qual seu grau de satisfação em relação ao material didático } \\
\text { disponibilizado/indicado pelos professores do Departamento de Estatística? }\end{array}$ \\
\hline Disponib_Outros & $\begin{array}{c}\text { Qual seu grau de satisfação em relação à disponibilidade fora de sala de aula dos } \\
\text { professores dos demais departamentos? }\end{array}$ \\
\hline Dominio_Outros & $\begin{array}{l}\text { Qual seu grau de satisfação em relação ao domínio do conteúdo pelos professores } \\
\text { dos demais departamentos? }\end{array}$ \\
\hline Pontual_Outros & $\begin{array}{l}\text { Qual seu grau de satisfação em relação à pontualidade dos professores dos demais } \\
\text { departamentos? }\end{array}$ \\
\hline Material_Outros & $\begin{array}{l}\text { Qual seu grau de satisfação em relação ao material didático } \\
\text { disponibilizado/indicado pelos professores dos demais departamentos? }\end{array}$ \\
\hline Pontual_Aluno & Qual seu grau de satisfação em relação à sua pontualidade? \\
\hline Estudo_Aluno & Qual seu grau de satisfação em relação ao seu comprometimento com os estudos? \\
\hline Prazos_Aluno & $\begin{array}{l}\text { Qual seu grau de satisfação em relação ao seu comprometimento com os prazos de } \\
\text { entrega de listas/trabalhos? }\end{array}$ \\
\hline Particip_Aluno & Qual seu grau de satisfação em relação à sua participação em sala de aula? \\
\hline
\end{tabular}

$$
K M O=\frac{\sum_{j=1}^{p} \sum_{m=1, m \neq j}^{p} r_{j m}^{2}}{\sum_{j=1}^{p} \sum_{m=1, m \neq j}^{p} r_{j m}^{2}+\sum_{j=1}^{p} \sum_{m=1, m \neq j}^{p} r_{p j m}^{2}}
$$

em que $r_{j m}^{2}$ é o coeficiente de correlação linear entre $X j \mathrm{e} X m ; r_{p j m}^{2}$ é o coeficiente de correlação parcial amostral entre Xje Xme definido como sendo o coeficiente de correlação linear entre os resíduos.

$\mathrm{O}$ KMO pode variar de 0 a 1 . Os valores iguais ou próximos a 0 indicam que a soma das correlações parciais dos itens avaliados é bastante alta em relação à soma das correlações totais. Nesses casos, possivelmente a análise fatorial será inapropriada (PASQUALI, 1999; DAMÁSIO, 2012). Valores menores que 0,5 são considerados inaceitáveis, e valores entre 0,5 e 0,7 são considerados medíocres; valores entre 0,7 e 0,8 são considerados bons; valores maiores que 0,8 e 0,9 são considerados ótimos e excelentes (HUTCHESON; SOFRONIOU, 1999). Sendo assim, a medida de adequação da amostra (MAA) aceitável é maior do que 0,5 (PEREIRA, 1999).

\section{Modelo teórico da análise fatorial}

O modelo da análise de fatores pode ser escrito como (GUTTMAN, 1954; JOHNSON; WICHERN, 1998):

$$
X_{i}=a_{i 1} F_{1}+a_{i 2} F_{2}+\cdots+a_{i m} F_{m}+e_{i}
$$


em que $X_{i}$ é o i-ésimo escore depois ter sido padronizado (média 0 e desvio-padrão 1 ), " $i=$ $1, \cdots, p$ "e $p$ é o número de variáveis; $a_{i 1}, a_{i 2}, \cdots, a_{i m}$ são as cargas dos fatores para o i-ésimo teste; $F_{1}, F_{2}, \cdots, F_{m}$ são $m$ fatores comuns não correlacionados, cada um com média $0 \mathrm{e}$ variância 1 e $e_{i}$ é um fator específico somente para o i-ésimo teste que é não correlacionado com qualquer dos fatores comuns e tem média zero.

Os $p$ valores observados $X_{p}$ são expressos em termos de $p+m$ variáveis aleatórias não observáveis $\left(F_{1}, F_{2}, \cdots, F_{m} ; \varepsilon_{1}, \varepsilon_{2}, \cdots, \varepsilon_{p}\right)$, característica que distingue o modelo fatorial do modelo de regressão múltipla, sendo que as variáveis independentes podem ser observadas, e cujas posições são ocupadas por F no modelo fatorial (MINGOTI, 2005).

Matricialmente temos (JOHNSON; WICHERN, 1998; NEISSE; HONGYU, 2016):

$$
\boldsymbol{X}_{(p \times 1)}=\boldsymbol{\Lambda}_{(p \times m)} \mathbf{F}_{(m \times 1)}+\boldsymbol{\varepsilon}_{(p \times 1)}
$$

Com o modelo AF:

$$
\begin{gathered}
\operatorname{Var}\left(X_{j}\right)=a_{j 1}^{2} \operatorname{Var}\left(F_{1}\right)+a_{j 2}^{2} \operatorname{Var}\left(F_{2}\right)+\cdots+a_{j m}^{2} \operatorname{Var}\left(F_{m}\right)+\operatorname{Var}\left(e_{j}\right) \\
=a_{j 1}^{2}+a_{j 2}^{2}+\cdots+a_{j m}^{2}+\operatorname{Var}\left(e_{j}\right)
\end{gathered}
$$

em que $a_{j 1}^{2}+a_{j 2}^{2}+\cdots+a_{j m}^{2}$ é chamada a comunalidade de $X_{j}$ (a parte da sua variância que é explicada pelos fatores comuns). A comunalidade não pode exceder 1 , é preciso que $-1 \leq$ $a_{i j} \leq+1$.

Pode também ser estabelecido que a correlação entre $X_{j}$ e $X_{j}$, seja:

$$
r_{j j^{\prime}}=a_{j 1} a_{j \prime 1}+a_{j 2} a_{j \prime 2}+\cdots+a_{j m} a_{j \prime m}
$$

Consequentemente, duas variáveis somente serão altamente correlacionadas se elas tiverem altas cargas no mesmo fator (NEISSE; HONGYU, 2016).

\section{RESULTADOS E DISCUSSÕES}

Foi realizado o teste KMO para verificar o quão adequada é a aplicação da AFE para o conjunto de dados. $\mathrm{O}$ valor de KMO foi de 0,70 , o que indica uma boa adequação dos dados a análise fatorial.

A Medida de Adequação da Amostra (MAA) (Tabela 1)foi considerada aceitável para cada item $(>0,5)$ e, portanto deve-se prosseguir com a AFE, sendo que o item relativo a pontualidade dos professores do Departamento de Estatística e ao material didático disponibilizado/indicado pelos professores de outros departamentos que ministram aula para os alunos de Estatística apresentaram as maiores medidas de adequação da amostra.

Tabela 1. MAA para cada item do questionário.

\begin{tabular}{c|c|c|c|c|c}
\hline Item & MAA & Item & MAA & Item & MAA \\
\hline Salas & 0,64 & Disponib_Est & 0,75 & Material_Outros & 0,81 \\
Laboratorio & 0,67 & Dominio_Est & 0,69 & Pontual_Aluno & 0,67 \\
Biblioteca & 0,64 & Pontual_Est & 0,82 & Estudo_Aluno & 0,55 \\
Centro & 0,64 & Mat_Est & 0,74 & Prazos_Aluno & 0,74 \\
Departamento & 0,78 & Disponib_Outros & 0,74 & Particip_Aluno & 0,62 \\
Recursos & 0,76 & Dominio_Outros & 0,58 & & \\
Bolsas & 0,62 & Pontual_Outros & 0,69 & & \\
\hline
\end{tabular}


O screeplotda Figura 1 foi obtido por meio de uma AFE, utilizando o método de extração componentes principais e método de rotação varimax, em uma matriz de correlação composta por 19 variáveis. Conforme se pode observar no gráfico, a retenção de fatores utilizando o critério de Kaiser-Guttman(autovalor > 1), se pode perceber que quatro fatores deveriam ser retidos, visto que os primeiros quatro fatores apresentaram autovalores maiores do que 1.

Figura 1. Gráfico screeplot dos autovalores obtidos na análise fatorial.

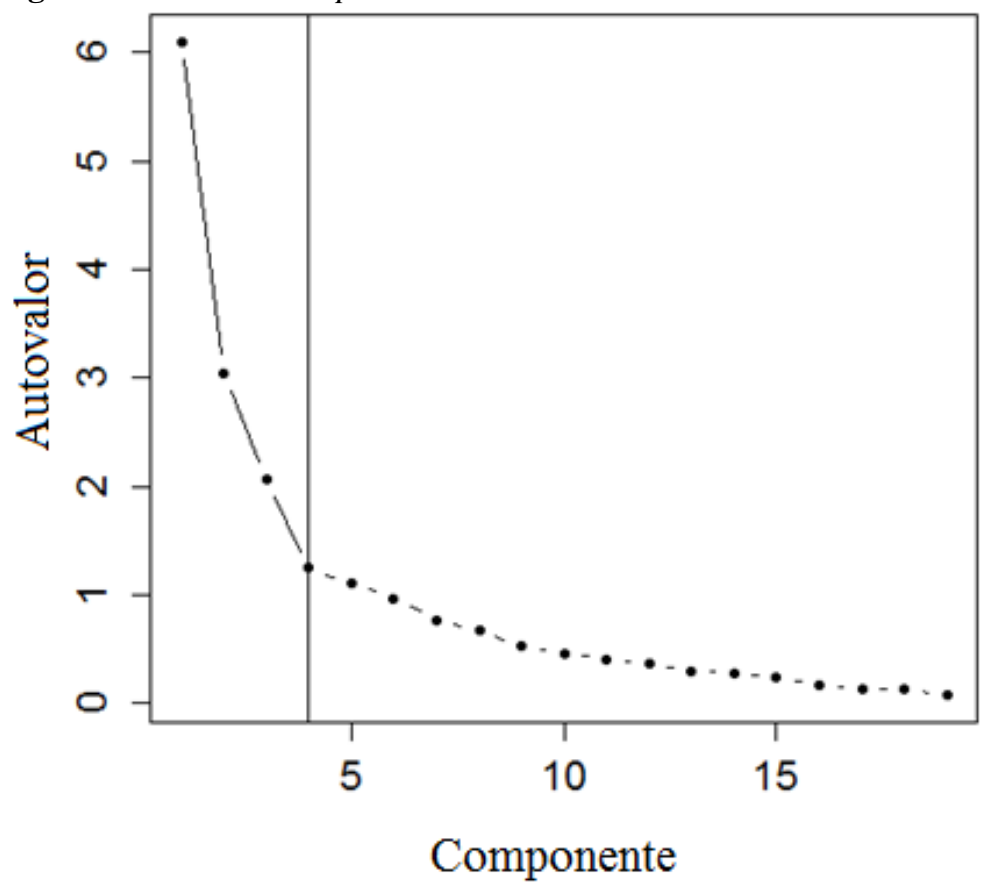

Para a realização da análise fatorial, foram determinados os autovalores e o percentual de variância explicada por meio de cada fator, como se pode visualizar na Tabela 2. O total dos fatores explicou $100 \%$ da variação total dos itens do questionário. O primeiro fator explicou $27 \%$ da variação total, o segundo, $26 \%$, o terceiro, $24 \%$, e o quarto, $23 \%$.

Tabela 2. Autovalores e percentual da variância explicada.

\begin{tabular}{c|c|c|c|c}
\hline Fator & Autovalor & $\begin{array}{c}\text { Autovalor } \\
\text { acumulado }\end{array}$ & $\begin{array}{c}\text { \% } \\
\text { Variância } \\
\text { Explicada }\end{array}$ & $\begin{array}{c}\text { \% Variância } \\
\text { Explicada } \\
\text { Acumulada }\end{array}$ \\
\hline 1 & 2,89 & 2,89 & 27 & 27 \\
2 & 2,86 & 5,75 & 26 & 53 \\
3 & 2,65 & 8,40 & 24 & 77 \\
4 & 2,51 & 10,91 & 23 & 100 \\
\hline
\end{tabular}

Na Figura 2 é apresentado o gráfico de correspondência para as cargas prévias. Observa-se que não é possível definir claramente as variáveis de cada fator sem a aplicação da rotação pelo método Varimax. Desta forma, procedeu-se a rotação Varimax, que maximiza a variância das cargas fatoriais para cada fator por meio do aumento das cargas altas e a diminuição das cargas baixas. Assim, observa-se pela Figura 3 que, após proceder-se a rotação Varimax, é possível identificar mais claramente quais itens correspondem a cada fator. 
Kirch, J.L.; Hongyu, K; Silva, F. de L;Dias, C. T.; Análise Fatorial para Avaliação dos Questionários de Satisfação do Curso de Estatística de uma Instituição Federal. E\&S - Engineering and Science, (2017), 6:1.

Figura 2. Representação gráfica dos planos fatoriais, Fator 1 versus o Fator 2 e não rotacionados em relação aos itens.

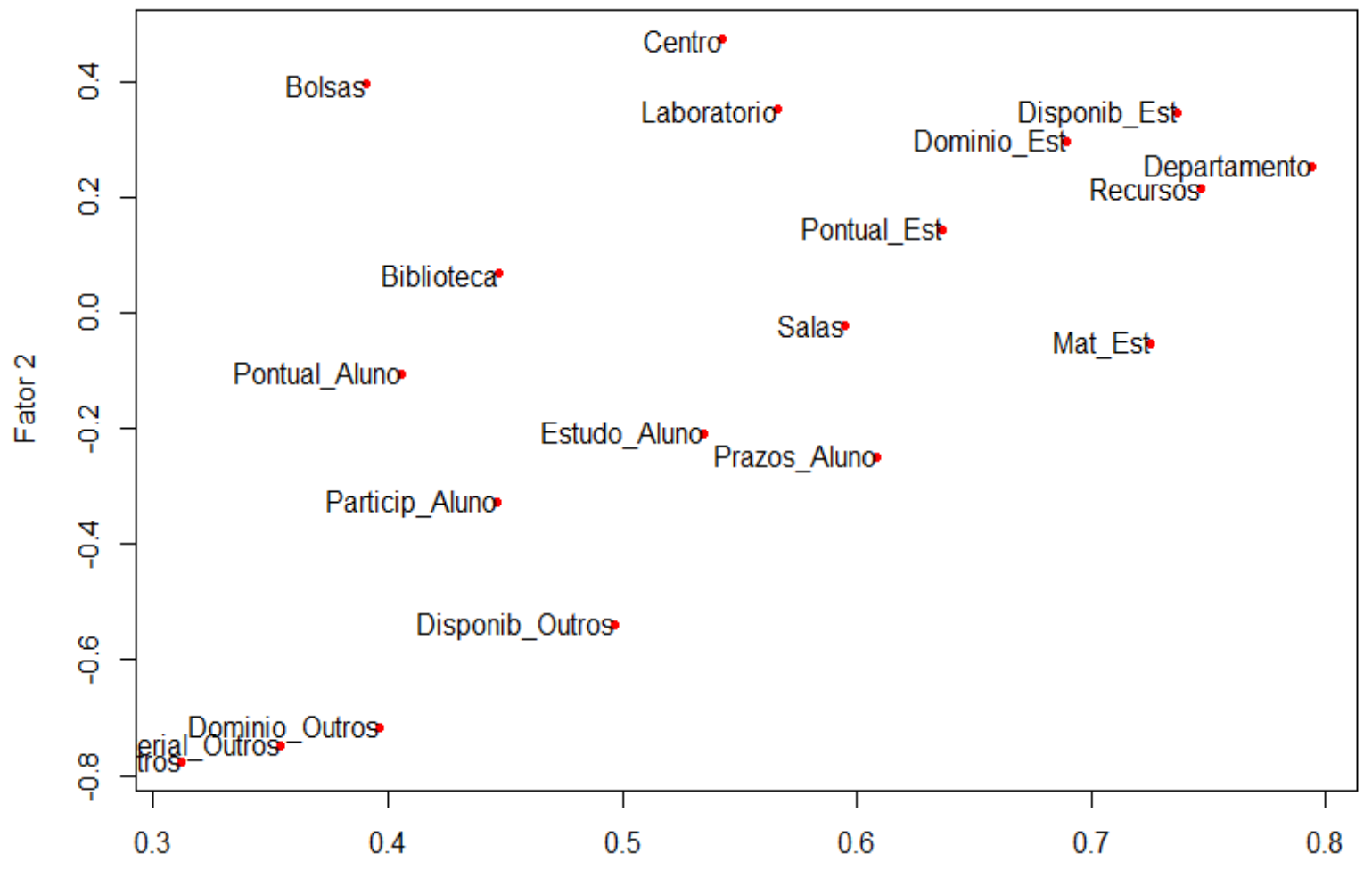

Fator 1

Figura 3. Representação gráfica dos planos fatoriais, Fator 1 versus o Fator 2 com método de rotação Varimax em relação aos itens.

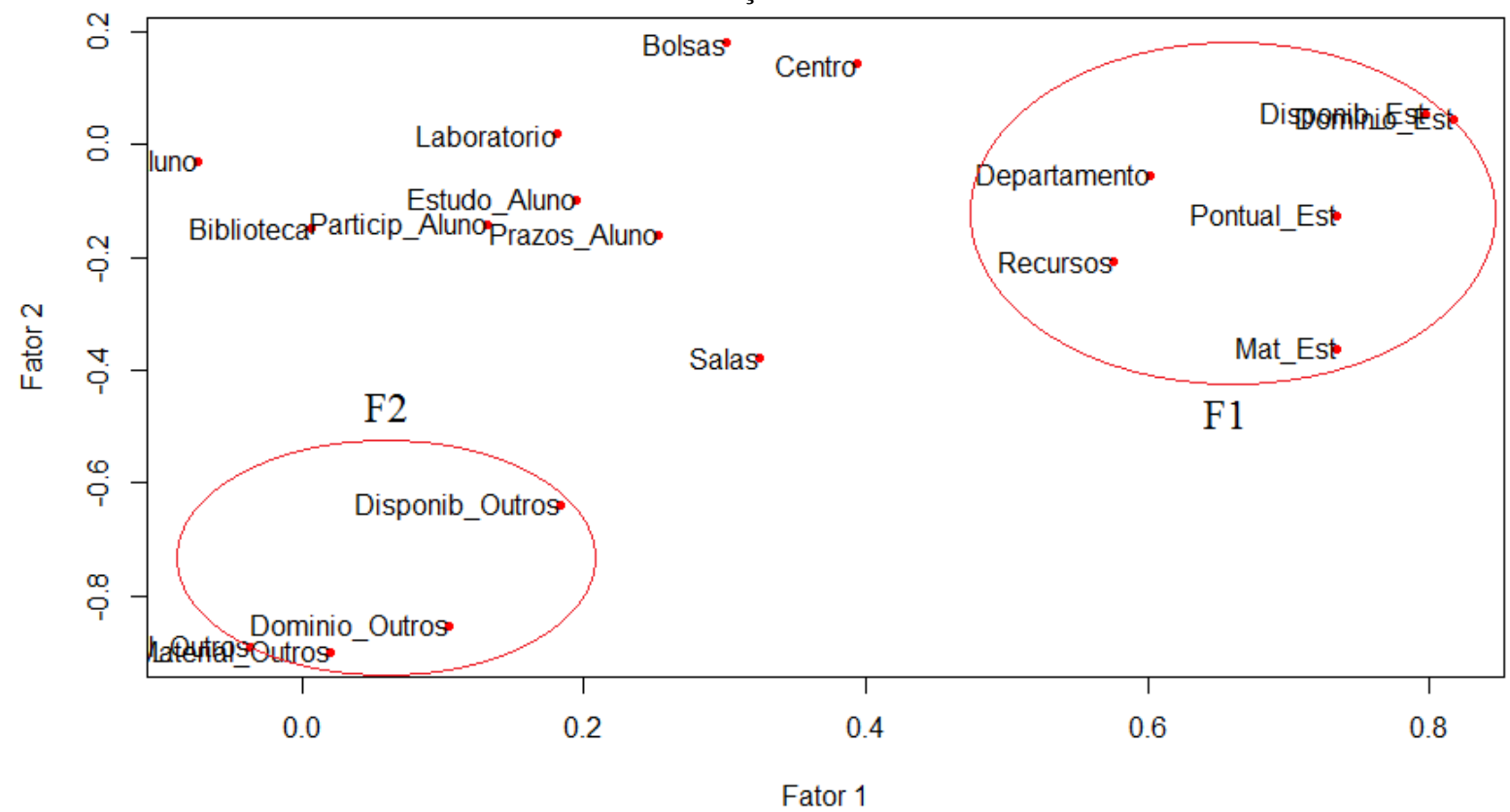

Na Tabela 3 estão descritos os valores das cargas fatoriais rotacionadas pelo método Varimaxdos quatro fatores para cada item do questionário e as suas comunalidades. Percebese que os itens Pontual_Outros $(0,89)$,Domínio_Outros $(0,86)$ e Material_Outros $(0,85)$ são os que apresentam maiores valores de cargas fatoriais para o Fator 1 - F1. Para o Fator 2 - F2, 
os maiores valores foram observados nos itens Laboratório $(0,77)$ e Recursos $(0,65)$. As maiores cargas fatoriais observadas para o Fator $3-\mathrm{F} 3$ foram encontradas nos itens Dominio_Est $(0,81)$ e Disponib_Est $(0,76)$. Para o Fator 4 - F4, os itens Prazos_Aluno $(0,84)$ e Estudo_Aluno $(0,83)$ foram os que apresentaram maiores valores de cargas fatoriais.

Tabela 3. Cargas fatoriaisrotacionadas pelo método Varimaxecomunalidade para cada item do questionário.

\begin{tabular}{c|c|c|c|c|c}
\hline Item & $\mathbf{F 1}$ & $\mathbf{F 2}$ & $\mathbf{F 3}$ & $\mathbf{F 4}$ & Comunalidade \\
\hline Salas & 0,31 & 0,62 & 0,13 & 0,05 & 0,50 \\
Laboratorio & $-0,06$ & $\mathbf{0 , 7 7}$ & 0,11 & 0,11 & 0,62 \\
Biblioteca & 0,09 & 0,41 & 0,12 & 0,15 & 0,22 \\
Centro & $-0,09$ & 0,56 & 0,35 & $-0,01$ & 0,44 \\
Departamento & 0,06 & 0,57 & 0,49 & 0,28 & 0,64 \\
Recursos & 0,18 & $\mathbf{0 , 6 5}$ & 0,44 & 0,07 & 0,65 \\
Bolsas & $-0,08$ & 0,40 & 0,22 & 0,04 & 0,22 \\
Disponib_Est & $-0,06$ & 0,39 & $\mathbf{0 , 7 6}$ & 0,17 & 0,76 \\
Dominio_Est & $-0,01$ & 0,24 & $\mathbf{0 , 8 1}$ & 0,16 & 0,74 \\
Pontual_Est & 0,15 & 0,34 & 0,49 & 0,14 & 0,40 \\
Mat_Est & 0,33 & 0,29 & 0,62 & 0,11 & 0,58 \\
Disponib_Outros & 0,54 & 0,09 & 0,23 & 0,26 & 0,42 \\
Dominio_Outros & $\mathbf{0 , 8 6}$ & $-0,01$ & 0,12 & 0,10 & 0,76 \\
Pontual_Outros & $\mathbf{0 , 8 9}$ & 0,03 & $-0,08$ & 0,08 & 0,81 \\
Material_Outros & $\mathbf{0 , 8 5}$ & 0,05 & 0,00 & 0,07 & 0,72 \\
Pontual_Aluno & 0,04 & 0,18 & 0,02 & 0,52 & 0,31 \\
Estudo_Aluno & 0,09 & 0,16 & 0,08 & $\mathbf{0 , 8 3}$ & 0,72 \\
Prazos_Aluno & 0,14 & 0,11 & 0,23 & $\mathbf{0 , 8 4}$ & 0,79 \\
Particip_Aluno & 0,17 & $-0,08$ & 0,17 & 0,74 & 0,62 \\
\hline
\end{tabular}

A Figura 4 apresenta o comportamento dos alunos em relação aos fatores. Observa-se que essa relação é mais dispersa entre os fatores 2 e 3 (F2 e F3). Para todas as outras relações, é possível observar um discreto agrupamento dos indivíduos, contendo alguns alunos que se distanciam do padrão observado para aquela relação.

Figura 4. Plano fatorial de correspondência dos 4 fatores em relação aos alunos do curso de Estatística.

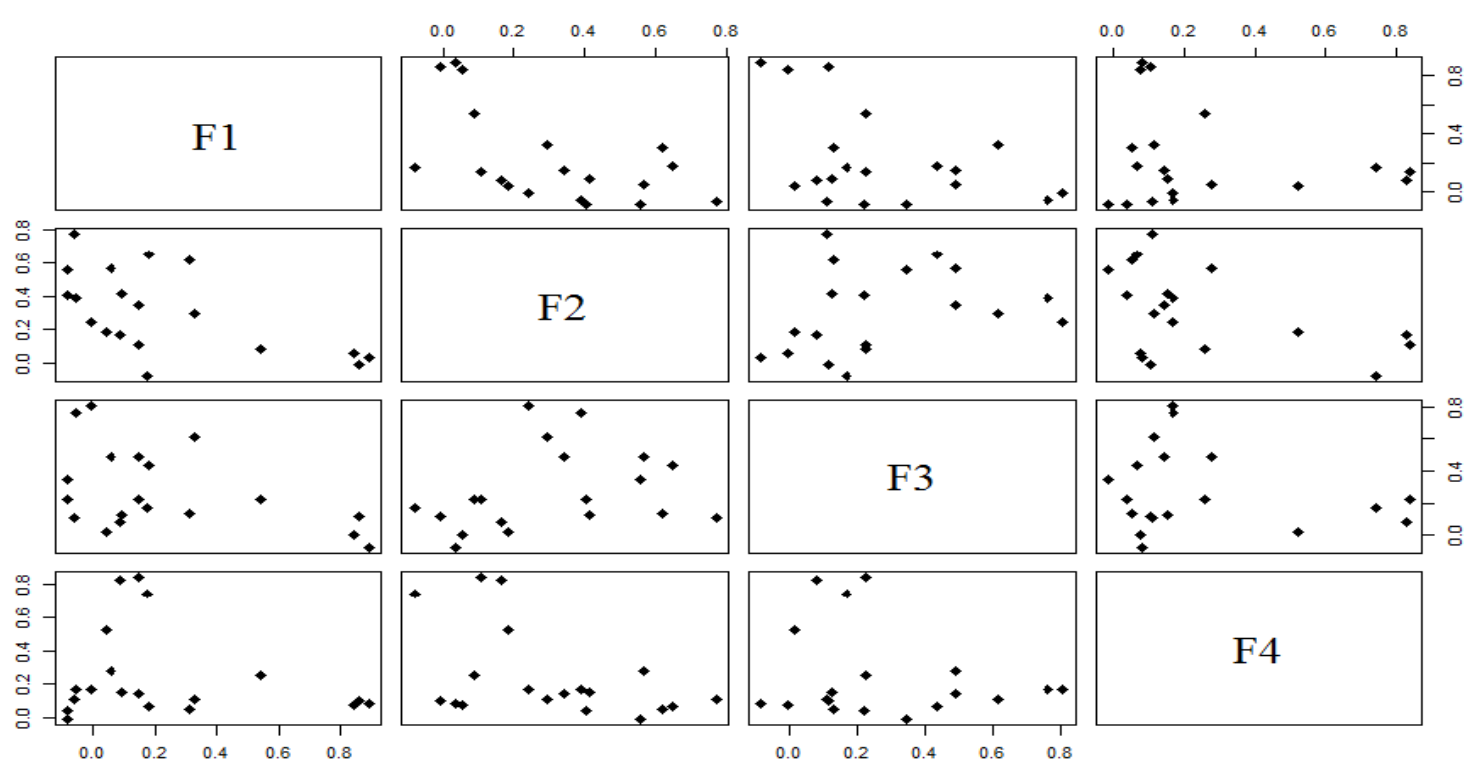


Figura 5. Itens que compõem os quatro fatores e suas respectivas cargas fatoriais.

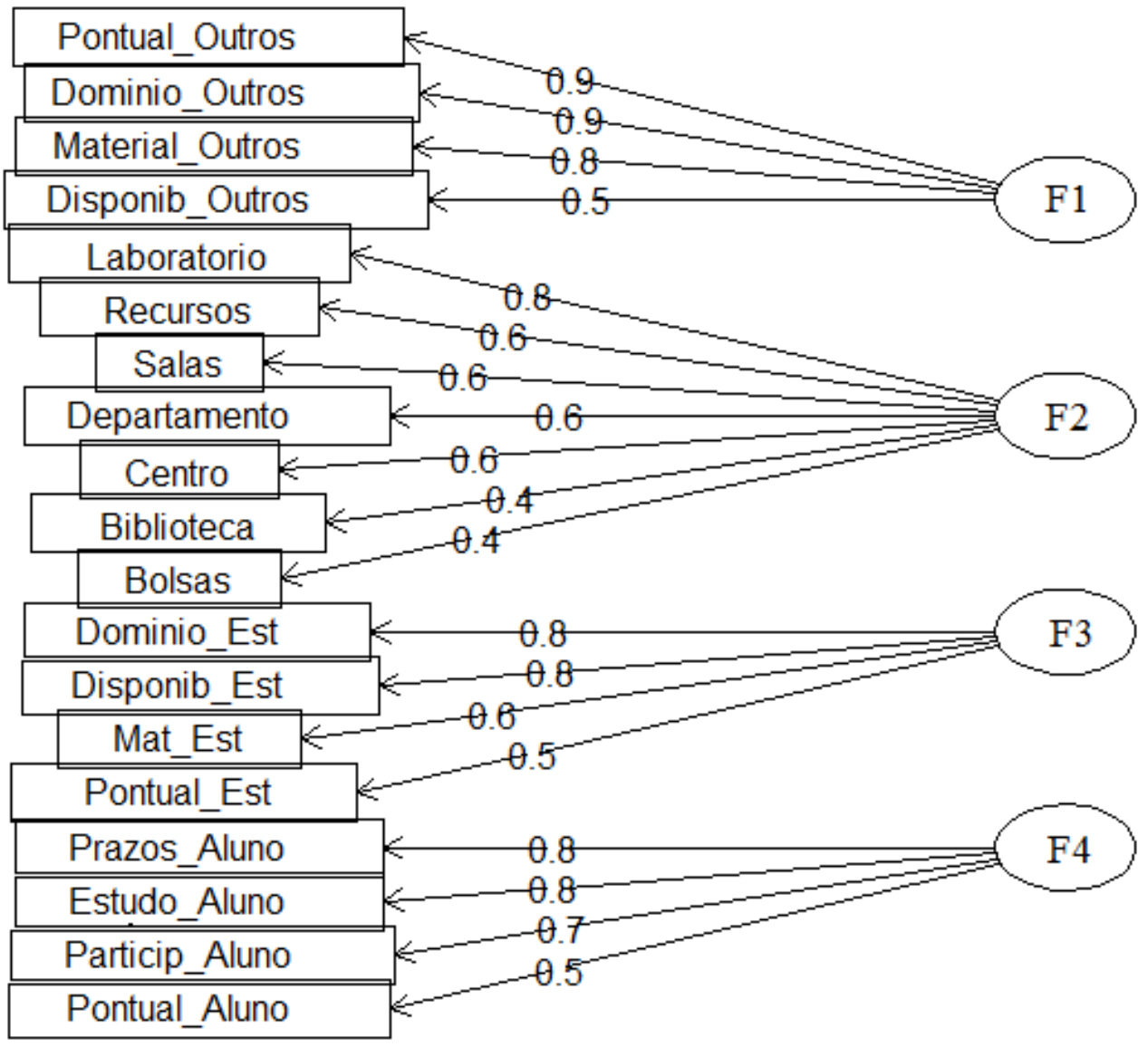

O primeiro fator (F1) agrupou os itens relacionados aos professores de outros departamentos que ministram aulas aos alunos de Estatística. Pode-se observar, pela Figura 5, que dos 4 itens que compõem o F1, todos possuem cargas fatoriais maiores que 0,5. Os itens pontualidade (Pontual_Outros) e domínio do conteúdo dos professores dos outros departamentos (Dominio_Outros) são os que apresentaram os maiores valores, sendo este igual a 0,9 cada. A disponibilidade fora de sala de aula dos professores dos outros departamentos apresentou o menor valor de carga fatorial para o fator, sendo igual a 0,5. Esse resultado pode ser confirmado pela Tabela 3, observando a comunalidade dos itens relativos ao F1.

$\mathrm{O}$ segundo fator (F2) agrupou os itens relacionados as instalações físicas e estruturais (Figura 5). O maior valor de carga fatorial foi observado para o item que mede a satisfação dos alunos em relação ao laboratório de Estatística. (Laboratório). Os itens biblioteca (Biblioteca) e disponibilidade de bolsas (Bolsas) apresentam as menores cargas fatoriais para o F2, sendo igual a 0,4 cada. As comunalidades para os itens desse fator são observadas na Tabela 3. Quanto maior a comunalidade, maior será o poder de explicação daquele item pelo modelo fatorial. Em geral, esperam-se comunalidades maiores que 0,5. Observou-se que a maior comunalidade foi encontrada para o item de satisfação quanto aos recursos tecnológicos (Recursos)

Os itens relacionados aos professores do Departamento de Estatística foram agrupados no terceiro fator (F3). Pode-se observar, ainda pela Figura 5, que os itens que compõem esse fator possuem cargas fatoriais maiores que 0,5. Os itens domínio do conteúdo (Dominio_Est) e disponibilidade fora de sala de aula dos professores do Departamento de Estatística 
(Disponib_Est) são os que apresentaram os maiores valores, sendo este igual a 0,8 cada. Esses itens também foram os que apresentaram as maiores comunalidades para o F3 (Tabela 3). A pontualidade dos professores do departamento do curso apresentou o menor valor de carga fatorial para o F3, sendo igual a 0,5 (Figura 5).

$\mathrm{O}$ quarto fator (F4) agrupou os itens relacionados ao desempenho do aluno, por meio da sua auto avaliação (Figura 5).Todos os itens que compreendem o F4apresentaram cargas fatoriais maiores que 0,5 . Os maiores valores de cargas fatoriais foram observados para os itens que medem a satisfação dos alunos em relação ao seu comprometimento com os prazos de entrega de listas/trabalhos (Prazos_Aluno), que apresentou a maior comunalidade para o F4 (Tabela 3), e ao seu comprometimento com os estudos (Estudo_Aluno). Os itens relativos à pontualidade do aluno (Pontual_Aluno) apresentou o menor valor de carga fatorial para o $\mathrm{F} 4$, sendo este igual a 0,5 .

\section{CONCLUSÃO}

A análise fatorial exploratória para o questionário de avaliação da satisfação dos alunos mostrou a formação de quatro grupos de variáveis. Para aplicações futuras, sugere-se a divisão do instrumento de avaliação da satisfação dos alunos em quatro grupos de questões, visto que as variáveis agruparam-se em quatro fatores: um dos grupos agregou as variáveis referentes aos professores de outros departamentos que ministram aula para os alunos da Estatística, o segundo grupo refere-se as instalações físicas e estruturais do curso de Estatística, incluindo neste grupo itens sobre a biblioteca, as salas de aula, o laboratório, o departamento, o centro acadêmico de Estatística, os recursos tecnológicos e a disponibilidade de bolsas de monitoria, PIBIC (Programa Institucional de Bolsas de Iniciação Científica), bolsas de extensão e outras. O terceiro grupo refere-se aos professores do Departamento de Estatística e o quarto grupo, à auto avaliação do aluno, que inclui itens sobre a satisfação do aluno em relação a sua própria pontualidade, ao seu comprometimento com os estudos, comprometimento com os prazos de entrega de listas/trabalhos e em relação à sua participação em sala de aula.

Este instrumento de avaliação de satisfação do aluno revelou ser um questionário com dimensões bem definidas. Os itens que compunham cada fator foram agrupados de maneira coerente com o assunto ao qual abordavam.

A análise desenvolvida neste estudo salienta a importância de pesquisas relativas a satisfação dos alunos de cursos de graduação, uma vez que o conhecimento do grau de satisfação dos alunos possibilita identificar as áreas em que a qualidade alcançada não atende as expectativas de qualidade dos alunos e a importância atribuída a cada item explorado, considerando para isso o papel do aluno na qualidade de um curso de educação superior.

\section{REFERÊNCIAS}

DZIUBAN, C.D. SHIRKEY, E,S. When is a correlation matrix appropriate for factor analysis? Some decision rules. Psychological Bulletin, 81(6), 358-361, 1974.

GUTTMAN, L. Some necessary conditions for common factor analysis, Psychometrika, 19, 149-162, 1954.

HAIR, J. F., ANDERSON, R. E., TATHAM, R. L. BLACK, W. C. Análise multivariada de dados. A. S. Sant'Anna \& A. C. Neto (Trad.). Porto Alegre: Bookman. 2005. 
HOSS, M., CATEN, C. S. Processo de Validação Interna de um Questionário em uma Survey Research Sobre ISO 9001:2000. Produto\&Produção, vol. 11, n. 2, p. 104 - 119, jun. 2010

HUTCHESON, G. D. SOFRONIOU, N. The multivariate social scientist: Introductory statistics using generalized linear models. London: Sage Publications. 1999.

JOHNSON, R.A.; WICHERN, D.W. Applied multivariate statistical analysis. Madison: Prentice Hall International, 1998. 816p.

LORENZO-SEVA, U. FERRANDO, P. J. FACTOR: A computer program to $\mathrm{ft}$ the exploratory factor analysis model. Behavior Research Methods, 38(1), 88-91. 2006.

MINGOTI, S. A. Análise de dados através de métodos de estatística multivariada: uma abordagem aplicada. Editora UFMG, 2005.

NEISSE, A. C. HONGYU, K. Aplicação de componentes principais e análise fatorial a dados criminais de 26 estados dos EUA. E\&S ENGINEERING AND SCIENCE. V. 5, N. 2. 2016.

PASQUALI, L. Análise fatorial: um manual teórico-prático. Brasília: Editora UnB. 1999.

PEREIRA, J, C, R. Análise de dados qualitativos: estratégias metodológicas para as ciências da saúde, humanas e sociais. São Paulo: EDUSP. 1999.

R DEVELOPMENT CORE TEAM. R: a language and environment for statistical computing. Vienna: R Foundation for Statistical Computing, Vienna, 2014. 